\title{
Epitope variation in the Newcastle disease virus $H N$ gene under antibody immune selective pressure in cell culture
}

\author{
GONG YanYan \& CUI ZhiZhong* \\ College of Animal Science and Veterinary Medicine, Shandong Agricultural University, Tai'an 271018, China
}

Received November 18, 2009; accepted November 9, 2010; published online March 31, 2011

\begin{abstract}
The influence of antibody immune selective pressure on Newcastle disease virus (NDV) $H N$ and $F$ gene mutations was studied in cell cultures. NDV field strain TZ060107 was inoculated into chicken embryo fibroblast cells and continuously passaged with (group A) or without (group B) anti-NDV monospecific serum. Each group contained three independent passage series. $H N$ and $F$ genes were amplified and sequenced for the 10th, 20th, 30th, 40th and 50th generations of each serial passage, and compared with the original strain. The results demonstrated that increased $H N$ gene mutations were observed in group A with the antibody than in group B without the antibody. The nonsynonymous (NS) to synonymous (S) mutations ratio was 6 for group A, significantly higher than 3.4 in group B. In group A with the antibody, there were five stable NS mutations in $H N$ gene, three of which (related to aa\#353, 521 and 568) were related to known epitopes. There were two stable NS mutations in $F$ gene in group A, but no stable NS mutations in group B. The NS/S ratios of $F$ gene were less than 2.5 for both groups A and B. Our results suggested that the antibody strongly influenced $H N$ gene mutations, while the $F$ gene was less influenced by the same antibody.
\end{abstract}

Newcastle disease virus, $H N$ gene, $F$ gene, immune selection, genetic variation

Citation: Gong Y Y, Cui Z Z. Epitope variation in the Newcastle disease virus $H N$ gene under antibody immune selective pressure in cell culture. Sci China Life Sci, 2011, 54: 474-479, doi: 10.1007/s11427-011-4161-2

Newcastle disease (ND) is an acute infectious disease in chickens caused by the avian paramyxovirus type 1 virus, Newcastle disease virus (NDV). It is one of the most serious diseases for economic losses in the poultry industry in China. NDV is an enveloped RNA virus with a single non-segmented negative strand. Its genome contains six genes encoding the nucleoprotein, phosphoprotein, matrix protein, fusion protein $(\mathrm{F})$, hemagglutinin-neuraminidase $(\mathrm{HN})$, and polymerase [1]. The capsule of NDV is composed of $\mathrm{F}$ and $\mathrm{HN}$ glycoproteins. F glycoprotein can fuse to host cell membrane and HN glycoprotein has hemagglutination and neuraminidase activities. Both $F$ and $H N$ are involved in cell recognition and attachment during infection, and are the main genes associated with NDV virulence [2-5].

*Corresponding author (email: zzcui@ @adu.edu.cn)
NDV has only one serotype, but it is divided into nine genotypes based on $F$ gene sequences. Each NDV genotype may exhibit differences in virulence and origin [6]. At present, genotype VII is the most prevalent NDV in China. Although most chicken farms have continuously conducted comprehensive vaccination programs against ND for many years in China, NDV infection is still common nationwide. Besides fatal acute or subacute cases, most NDV infections cause serious reductions in egg production and subsequent economic losses. It has been speculated that antigenic variation occurs within NDV field strains and was responsible for vaccination failures, although evidence of this remains inconclusive.

In recent years, our laboratory has studied the evolution of the $g p 85$ gene in the subgroup $\mathrm{J}$ avian leukosis virus and the hemagglutinin gene in the H9N2 subtype avian influ- 
enza virus under antibody selective pressure [7,8]. This study attempts to use similar experimental methodology to understand if mutations in NDV $H N$ and $F$ genes could be increased under the selective pressure of anti-NDV antibodies.

\section{Materials and methods}

\subsection{Virus}

NDV field strain TZ060107 (genotype VII) was used in this study. The virus was isolated from chicken embryos from a breeding farm, with reduced egg production, in 2006 [9].

\subsection{Preparation of cell cultures}

Chicken embryo fibroblast cells (CEF) were prepared from 10-day-old specific pathogen free (SPF) chicken embryos and used for NDV passage.

\subsection{Preparation of anti-NDV chicken serum and de- termination of its optimal dilutions for cell cultures}

Eight 21-day-old SPF chickens were intramuscularly inoculated with $0.5 \mathrm{~mL}$ of inactivated NDV strain TZ060107 vaccine in an oil emulsion. SPF chickens were raised in an isolator with positively pressured filtered-air. Chickens were vaccinated again two weeks later. Ten days after the second vaccination, chickens were bled and serum samples were collected as antiserum against NDV strain TZ060107. The hemagglutination inhibition titers (HI) ranged from $2^{8}$ to $2^{10}$. All serum samples were combined in a centrifuge tube and spun at $10000 \mathrm{r} \mathrm{min}^{-1}$. Serum was then filtered through a $0.22 \mu \mathrm{m}$ filter and inactivated at $56^{\circ} \mathrm{C}$ for $30 \mathrm{~min}$ and stored at $-20^{\circ} \mathrm{C}$ before use.

The original NDV strain TZ060107 was titrated in 96-well plates with CEF and the virus stock TCID $_{50}$ was calculated according to the Reed-Muench method. The primary virus neutralization test was conducted to determine the optimal dilution of the antiserum for the subsequent serial passages. It was carried out using virus of a fixed dilution and serum of different dilutions in a six-well plate containing CEF monolayers. Two control wells were used. One well contained the virus only and the other contained the antiserum only. The other four wells contained mixtures of the virus and antiserum at $1: 10,1: 20,1: 50$ and $1: 100$ dilutions. Plates were incubated for $72 \mathrm{~h}$ at $37^{\circ} \mathrm{C}$ with $5 \%$ $\mathrm{CO}_{2}$. CEF monolayers were predicted to be normal in the serum only control well and reduced, with rounded cells, in the virus only control well. The virus cytopathic effect (CPE) was observed and compared in the four left wells. The lowest serum dilution used in wells that demonstrated CPE was chosen for the following passages.

\subsection{Serial passages of NDV strain TZ060107 in CEF}

CEF monolayers for NDV strain TZ060107 inoculation were prepared and maintained in culture medium with $1 \%$ fetal bovine serum in six-well plates. Wells for each passage were divided into groups A and B. Wells for group A were supplemented with anti-TZ060107 chicken serum at the specific dilution determined in 1.3. Wells for group B without anti-TZ060107 serum were used as controls. An identical amount of NDV from the same stock of strain TZ060107 was inoculated into each well. In the first passage, three wells were designated as lineages A1-1, A2-1 and A3-1 for group A and B1-1, B2-1 and B3-1 for group B. When $50 \%$ of the monolayer cells demonstrated CPE, $100 \mu \mathrm{L}$ of supernatant from each well was collected and inoculated separately into another six-well plate with fresh CEF monolayers. The six wells in the second plate were also divided as described above; however, wells were designated as lineages A1-2, A2-2 and A3-2 for group A and B1-2, B2-2, and B3-2 for group B for the second passages. These steps were repeated for 50 passages. Every 10 passages, the supernatants in each well of the six lineages were harvested for the viruses and stored at $-70^{\circ} \mathrm{C}$.

\subsection{Amplification, cloning and sequencing of $H N$ and $F$ genes}

Whole genomic RNA in the supernatants from each well of the A1-A3 and B1-B3 lineages at the 10th, 20th, 30th, 40th and 50th passages was extracted using TRizol reagent (Invitrogen, Cat No. 15596-026) according to the manufacturer's instructions. Primers for $F$ gene amplification $(1725$ bp) were $F_{1}: 5^{\prime}$-GGCAAATTAGAAAAAACACGGG-3' and $F_{2}$ : 5'-CTCTTGTAGTGGCTCTCATCT-3'; primers for $H N$ gene amplification (1801 bp) were $H N_{1}$ : 5'-TCCGTTCTACCAATCACCA-3' and $\mathrm{HN}_{2}$ : 5'-CGTCTTCCCAACCATCCTAT-3'. Primers were synthesized by Shanghai Sangon Co., Ltd., China.

Reverse transcription-polymerase chain reaction (RTPCR) amplification was conducted using a TaKaRa RNA PCR Kit (AMV) Ver.3.0 (TaKaRa Biotechnology Co., Ltd.) according to the manufacturer's instructions. RT-PCR reaction conditions for the $H N$ gene were $95^{\circ} \mathrm{C}$ denaturation for $6 \mathrm{~min}, 95^{\circ} \mathrm{C}$ for $1 \mathrm{~min}, 54^{\circ} \mathrm{C}$ for $1 \mathrm{~min}$ and $72^{\circ} \mathrm{C}$ for $2 \mathrm{~min}$ for a total of 32 cycles, $72^{\circ} \mathrm{C}$ extension for $10 \mathrm{~min}$ and $4^{\circ} \mathrm{C}$ to terminate the reaction. RT-PCR reaction conditions for the $F$ gene were $95^{\circ} \mathrm{C}$ denaturation for $6 \mathrm{~min}, 95^{\circ} \mathrm{C}$ for 1 $\min , 54^{\circ} \mathrm{C}$ for $1 \mathrm{~min}$ and $72^{\circ} \mathrm{C}$ for $1 \mathrm{~min} 45 \mathrm{~s}$ for a total of 32 cycles, $72^{\circ} \mathrm{C}$ extension for $10 \mathrm{~min}$ and $4^{\circ} \mathrm{C}$ to terminate the reaction.

Cloning, identification and sequencing of the $H N$ and $F$ genes were conducted by cloning PCR products into pMD18-T and sequencing using commercial services. 
1.6 Analysis of ratios of non-synonymous and synonymous mutations in $H N$ and $F$ gene sequences

DNA Star software was used for analysis and comparison. Non-synonymous (NS) and synonymous (S) mutation ratios (NS/S value) were analyzed and compared according to published methods [10].

\section{Results}

2.1 Determination of the highest anti-NDV serum concentration required for partial virus replication inhibition

Virus neutralization indicated that virus replication was completely inhibited in media with anti-NDV chicken serum at a 1:20 dilution. However, viruses could still replicate at different levels in media with identical serum at 1:50 and $1: 100$ dilutions. Therefore, anti-NDV chicken serum at a 1:50 dilution was used for the subsequent 50 serial passages of the A1, A2 and A3 lineages.

\subsection{Stability of $H N$ and $F$ gene sequences in different passages}

Sequence comparisons showed that the amplified $H N$ gene fragments were composed of $1801 \mathrm{bp}$ and contained a complete open reading frame $(1716 \mathrm{bp})$ in all passages. The $F$ gene fragments were composed of $1725 \mathrm{bp}$ and contained a complete open reading frame $(1662 \mathrm{bp})$. After 50 passages, different levels of variation were detected in different passage serials, although all viruses maintained a 99\% homology to the original virus (GenBank accession No. FJ011444.1 and FJ011448.1).

\subsection{Variations during the serial passages with anti- NDV serum}

In group A, 67 different base mutations were observed in the $H N$ gene. Among them, 61 were non-synonymous mutations located at 30 different sites and six were synonymous in six different sites. A total of 38 mutations were observed in the $F$ gene, 24 of them were non-synonymous mutations located at 15 different sites and 14 were synonymous mutations at 11 different sites. These results revealed that the NS/S ratio for the $H N$ gene in group A was 30/6 (5), while the NS/S ratio for the $F$ gene in group A was only 15/11 (1.36).

Stable mutations mainly occurred during the 20th-50th passages and the mutated sites usually became stable after 30 passages (Table 1). Five mutation sites in the $H N$ gene were stable in more than two consecutive passages. The NS/S ratio in these five sites was 5/0, demonstrating the immune selective pressure effect of the anti-NDV antibody.
Among these five sites, two had the same mutation in two separate lineages. Another two mutations at base \#1439 and \#1703 were identical in the three independent lineages A1, A2 and A3 (Table 1). Stable mutations in at least two consecutive passages were found at three sites in the $F$ gene, with a NS/S ratio of $2 / 1$ (2).

\subsection{Variations during the serial passages without anti-NDV serum}

NDV TZ060107 strain was also continuously passaged in $\mathrm{CEF}$ without antibodies, and a total 24 mutations occurred in the $H N$ gene at 19 different sites. Among them, 20 were NS mutations found at 15 sites. Only one stable NS mutation occurred in more than two consecutive passages in one serial passage (Table 1). A further four $\mathrm{S}$ mutations were located at four sites, with a NS/S ratio of $15 / 4$ (3.75). A total of 17 mutations occurred in the $F$ gene at 15 sites, of which, nine were NS mutations located in eight sites and eight were $\mathrm{S}$ mutations located at seven sites, with a NS/S ratio of $8 / 7$ (1.14). Overall, the $F$ gene nucleotide variation was a result of random mutation and no stable mutations occurred in two consecutive passages.

\subsection{Influence of the antibody on the ratios of NS/S mutations of $F$ and $H N$ genes during passages}

The NS/S ratios for the $H N$ and $F$ genes in both groups A and $\mathrm{B}$ are listed in Table 2 . There were more NS mutations and higher NS/S ratios for the group A $H N$ gene than group B. However, such differences between groups were not as large for the $F$ gene when compared to the $H N$ gene, indicating that antibody-mediated immune selective pressure was higher for the $H N$ gene than for the $F$ gene.

\section{Discussion}

During interaction with the host, viruses continue to produce genetic mutations via various evolutionary processes. Recently, much attention has been focused on the influence of immune selective pressures in virus evolution. For example, human immunodeficiency virus (HIV) envelope glycoprotein genes were reported to have a high mutation frequency, and their 3rd hr-region (V3 loop) can have mutation rates as high as 50\% [11]. The V3 loop of HIV1 was closely related to its cell tropism, replication kinetics and cellular pathogenicity, and the mutations in the V3 loop could potentially help viruses escape attacks by cytotoxic $\mathrm{T}$ cells or neutralizing antibodies [12,13]. Hemagglutinin glycoprotein of human respiratory measles virus may also develop changes in its antigenicity, which helps the virus escape from host immune reaction, because of the influence of immune selective pressures [14]. These studies were based on the analysis of a large number of nucleotide sequences for 
Table 1 Stable mutation sites in the $H N$ and $F$ genes ORF during continued passages in $C_{E F}{ }^{\text {a) }}$

\begin{tabular}{|c|c|c|c|c|c|c|c|c|c|}
\hline \multirow{2}{*}{\multicolumn{2}{|c|}{ NS serial passages }} & \multicolumn{6}{|c|}{$H N$ gene } & \multicolumn{2}{|c|}{$F$ gene } \\
\hline & & $\begin{array}{l}\# 140 \\
(47)^{*}\end{array}$ & $\begin{array}{l}\text { \#776 } \\
(259)^{*}\end{array}$ & $\# 1058$ & $\begin{array}{l}\# 1439 \\
(480)^{*}\end{array}$ & $\# 1563$ & $\begin{array}{l}\# 1703 \\
(568)^{*}\end{array}$ & \#676 & $\# 1348$ \\
\hline \multirow{6}{*}{$\mathrm{A} 1$} & & & & & & & & & \\
\hline & A1-10 & - & - & - & - & - & - & - & - \\
\hline & A $1-20$ & - & - & - & - & - & - & - & - \\
\hline & A1-30 & - & $\mathrm{G}(\mathrm{G})$ & - & $\mathrm{A}(\mathrm{K})$ & - & $\mathrm{G}(\mathrm{G})$ & $\mathrm{G}(\mathrm{A})$ & - \\
\hline & A $1-40$ & - & $\mathrm{G}(\mathrm{G})$ & $\mathrm{A}(\mathrm{Q})$ & $\mathrm{A}(\mathrm{K})$ & - & $\mathrm{G}(\mathrm{G})$ & $\mathrm{G}(\mathrm{A})$ & - \\
\hline & A $1-50$ & - & $\mathrm{G}(\mathrm{G})$ & $\mathrm{A}(\mathrm{Q})$ & $\mathrm{A}(\mathrm{K})$ & - & $\mathrm{G}(\mathrm{G})$ & $\mathrm{G}(\mathrm{A})$ & - \\
\hline \multirow{5}{*}{ A2 } & A2-10 & - & - & - & - & - & - & - & - \\
\hline & A2-20 & - & - & - & - & - & $\mathrm{G}(\mathrm{G})$ & $\mathrm{G}(\mathrm{A})$ & - \\
\hline & A2-30 & - & $\mathrm{G}(\mathrm{G})$ & - & $\mathrm{A}(\mathrm{K})$ & - & $\mathrm{G}(\mathrm{G})$ & $\mathrm{G}(\mathrm{A})$ & - \\
\hline & A2-40 & - & $\mathrm{G}(\mathrm{G})$ & $\mathrm{A}(\mathrm{Q})$ & $\mathrm{A}(\mathrm{K})$ & - & $\mathrm{G}(\mathrm{G})$ & $\mathrm{G}(\mathrm{A})$ & - \\
\hline & A2-50 & - & $\mathrm{G}(\mathrm{G})$ & $\mathrm{A}(\mathrm{Q})$ & $\mathrm{A}(\mathrm{K})$ & - & $\mathrm{G}(\mathrm{G})$ & $\mathrm{G}(\mathrm{A})$ & - \\
\hline \multirow{5}{*}{ A3 } & A3-10 & - & - & - & - & - & $\mathrm{G}(\mathrm{G})$ & - & - \\
\hline & A3-20 & - & - & - & - & $\mathrm{A}(\mathrm{R})$ & $\mathrm{G}(\mathrm{G})$ & - & - \\
\hline & A3-30 & - & - & - & $\mathrm{A}(\mathrm{K})$ & $\mathrm{A}(\mathrm{R})$ & $\mathrm{G}(\mathrm{G})$ & - & $\mathrm{G}(\mathrm{V})$ \\
\hline & A3-40 & - & - & - & $\mathrm{A}(\mathrm{K})$ & $\mathrm{A}(\mathrm{R})$ & $\mathrm{G}(\mathrm{G})$ & - & $\mathrm{G}(\mathrm{V})$ \\
\hline & A3-50 & - & - & - & $\mathrm{A}(\mathrm{K})$ & $\mathrm{A}(\mathrm{R})$ & $\mathrm{G}(\mathrm{G})$ & - & $\mathrm{G}(\mathrm{V})$ \\
\hline \multirow{5}{*}{ B1 } & B1-10 & - & - & - & - & - & - & - & - \\
\hline & B1-20 & - & - & - & - & - & - & - & - \\
\hline & B1-30 & $\mathrm{A}(\mathrm{N})$ & - & - & - & - & - & - & - \\
\hline & B1-40 & $\mathrm{A}(\mathrm{N})$ & - & - & - & - & - & - & - \\
\hline & B1-50 & $\mathrm{A}(\mathrm{N})$ & - & - & - & - & - & - & - \\
\hline \multirow{5}{*}{ B2 } & B2-10 & - & - & - & - & - & - & - & - \\
\hline & B2-20 & - & - & - & - & - & - & - & - \\
\hline & B2-30 & - & - & - & - & - & - & - & - \\
\hline & B2-40 & $\mathrm{A}(\mathrm{N})$ & - & - & - & - & - & - & - \\
\hline & B2-50 & $\mathrm{A}(\mathrm{N})$ & - & - & - & - & - & - & - \\
\hline \multirow{5}{*}{ B3 } & B3-10 & - & - & - & - & - & - & - & - \\
\hline & B3-20 & - & - & - & - & - & - & - & - \\
\hline & B3-30 & $\mathrm{A}(\mathrm{N})$ & - & - & - & - & - & - & - \\
\hline & B3-40 & - & - & - & - & - & - & - & - \\
\hline & B3-50 & - & - & - & - & - & - & - & - \\
\hline
\end{tabular}

a) Numbers with "*” indicate non-synonymous mutation sites and "-" indicates identical bases and amino acids as the original strain. One stable synonymous mutation in $F$ gene was not included. Lineages A1-A3 were passaged in CEF with antibodies and B1-B3 were passaged in CEF without antibodies.

Table 2 Comparisons for the NS/S ratios for $H N$ and $F$ gene mutations between passages in CEF with or without anti-NDV serum ${ }^{\text {a) }}$

\begin{tabular}{|c|c|c|c|c|c|c|c|c|c|c|c|c|c|c|c|c|}
\hline \multirow{2}{*}{$\begin{array}{c}\text { Genes } \\
\text { Serial passages }\end{array}$} & \multicolumn{8}{|c|}{$H N$ gene } & \multicolumn{8}{|c|}{$F$ gene } \\
\hline & A1 & A2 & A3 & Total & B1 & B2 & B3 & Total & A1 & A2 & A3 & Total & B1 & B2 & B3 & Total \\
\hline NS sites & 11 & 12 & 13 & 36 & 6 & 4 & 7 & 17 & 6 & 3 & 8 & 17 & 2 & 4 & 3 & 9 \\
\hline $\mathrm{S}$ sites & 2 & 3 & 1 & 6 & 1 & 3 & 1 & 5 & 4 & 2 & 5 & 11 & 1 & 5 & 2 & 8 \\
\hline $\mathrm{NS} / \mathrm{S}$ & 5.5 & 4 & 13 & 6 & 6 & 1.33 & 7 & 3.4 & 1.5 & 1.5 & 1.6 & 1.55 & 2 & 0.8 & 1.5 & 1.125 \\
\hline
\end{tabular}

a) NS, non-synonymous; S, synonymous. Numbers indicate mutation sites detected during all passages, including both stable and instable mutations.

different strains, and were assessed on the basis of NS/S ratios for the associated genes. In general, when the NS/S ratio is higher than 2.5 , a selective pressure is present, such as the immune selective pressure. When a sufficient number of strains are compared, the higher the NS/S ratio is, the greater the role of a selective pressure [10]. In recent years, our laboratory, along with other research groups, have studied the influence of the immune selective pressure on the evolution of chicken leukemia virus [7], NDV attenuated vaccine [15], and H9N2 subtype avian influenza virus [8] in cell culture or embryonated eggs. As a continuation of the above work, this paper focused on the evolution of the NDV TZ060107 field strain during passages in CEF with NDV-specific antibodies.

In group A, the majority of NS mutations appeared in the 10th-30th passages and continued to be maintained in fur- 
ther passages. Some sites of NS mutations also occurred simultaneously in two or even three different passage lineages (Table 1). This indicates that such variation is not random but is instead strongly influenced by antibody immune selective pressure, or that these mutated viruses were originally a minority quasispecies that became dominant populations during passage. By contrast, most mutations in group $\mathrm{B}$ were located randomly at various sites for different passage generations in different passage lineages. These changes were less stable and were likely to be random mutations. $H N$ gene mutations in group A with antibody were apparently more common than in group B without antibody (Table 2). The NS/S mutation ratio for group A was 6, indicating a strong specific antibody selective pressure. For group B without antibody, there was only one stable NS mutation in the $H N$ gene and the average NS/S ratio was 3.4, which was significantly smaller than group A. The stable mutations observed in group B may relate to its adaptation in chicken embryo fibroblast, another kind of selective pressure.

Double selective pressures potentially existed in group A. There were five NS mutations in three passage lineages, which first occurred in the 10th-40th generations and remained stable up to the 50th generation (Table 1). However, mutations at the same nucleotide positions were not observed in any of the group B lineages, suggesting that mutations of these sites were associated with the presence of antibodies. There were also two stable NS mutations in the $F$ gene of group A. Stable NS mutations of $F$ gene were absent in group B. NS/S ratios of base mutations in the $F$ gene were less than 2.5 for both groups, suggesting that the effects of the two pressures on the $F$ gene were not as strong as on $H N$ (Table 2). This indicates that the process of NDV genetic evolution in the $H N$ and $F$ genes is relatively independent of each other [16]. Studies on the molecular epidemiology of the $H N$ and $F$ genes of NDV strains showed that over the last decade these genes in NDV field strains have deviated from those of the widely used vaccine virus LaSota strain, and that the $H N$ gene variation was greater than that of the $F$ gene [16]. This also suggests that antibodies strongly influence mutations in $H N$ gene rather than in $F$ gene.

Because of the lack of corrective function for RNA polymerase, RNA is prone to errors during replication. When amino acid mutations accumulate or cause epitopic variation, changes in antigenicity can occur. In cell cultures and particularly in vivo, virus populations can consist of quasispecies that exhibit specific genomic variations in each virus particle. If a virus with a particular mutation has a selective advantage in replication compared to the original genetic majority, it will grow faster and become increasingly dominant in the virus population. Otherwise, it will remain a genetic minority or even gradually disappear from the population if this mutation is disadvantageous. NDV mutations can produce epitopic changes that are no longer recognized by the original virus-specific antibodies. Such mutated viruses may subsequently become prevalent during passage with specific antibodies.

In our study, genetic mutations predominantly occurred in the 20th-30th generations. Viruses became stable in the 40th generation in group A, indicating that the specific immune pressure to the mutated virus was no longer effective. Our previous study on avian leukosis virus (subgroup J) found that amino acid mutations were mainly in the gp85 hypervariable region under antibody selective pressure [7], and that relevant mutations can occur simultaneously in 2-3 linked amino acids. However, mutations of H9N2-AIV hemagglutinin gene, under antibody selective pressure, were randomly distributed, which was consistent with the absence of a hypervariable region in this gene [8]. In our current study, amino acid mutations of the $H N$ and $F$ genes under antibody selective pressures were distributed across different regions, which is also consistent with no high variable regions having been found in the $H N$ and $F$ genes of NDV. However, three of five NS mutations in the $H N$ gene that were related to immune selective pressures (aa\#353, 521 and 568; Table 1) were located exactly within three known antigenic epitopes: aa\#345-353, 513-521, and $569[17,18]$. This also verifies that the three reported epitopes relate to virus neutralization, and provides direct evidence that these mutations are indeed associated with the antibody selective pressures.

Antigenicity of the HN protein is not only determined by the epitope regions, but also by the protein maturity and molecule conformation [19]. The non-continuous region of the molecule may form such $\mathrm{HN}$ antigenic sites in the natural protein structure. In addition, there are 12 conserved cysteine residues in HN peptide, located at aa\#172, 186, 196, $238,247,251,344,455,461,465,531$ and 542, which play an important role in the $\mathrm{HN}$ maturation and the maintenance of its three-dimensional structure. Specific residue mutations could potentially block the formation of the corresponding antigenic site [20]. Such cysteine residues did not mutate in both groups A and B in our study, indicating that mutations under immune selective pressure only occur in given epitopes, rather than the entire three-dimensional structure of the molecule.

This work was supported by the Shandong Province Application Technology Innovation Project on Agriculture during 2007 and 2008.

1 Calnek B W, Barnes H J, Beard C W, et al. Disease of Poultry. 10th ed. Iowa: Iowa State University Press, 1997. 541-562

2 Aldous E W, Alexander D J. Detection and differentiation of Newcastle disease virus (avian paramyxovirus type 1). Avain Pathol, 2001, 30: $117-128$

3 Panda A, Huang Z, Elankumaran S, et al. Role of fusion protein cleavage site in the virulence of Newcastle disease virus. Microb Pathog, 2004, 36: 1-10

4 Nagai Y, Klenk H D, Rott R. Proteolytic cleavage of the viral glycoproteins and its significance for the virulence of Newcastle disease 
virus. Virology, 1976, 72: 494-508

5 Peeters B P, De Leeuw O S, Koch G, et al. Rescue of Newcastle disease virus from cloned cDNA: Evidence that cleavability of the fusion protein is a major determinant for virulence. J Virol, 1999, 73: 5001-5009

6 Liu X F, Wan H Q, Ni X X, et al. Pathotypical and genotypical characterization of strains of Newcastle Disease virus isolated from outbreaks in chicken and goose in some regions of China during 1985-2001. Arch Virol, 2003, 148: 1387-1403

7 Wang Z F, Cui Z Z. Evolution of gp85 gene of subgroup J avian leukosis virus under the selective pressure of antibodies. Sci China Ser C-Life Sci, 2006, 49: 1-8

8 Lou B H, Zhu X T, Cui Z Z, et al. Mutations of the hemagglutinin gene of H9N2 subtype avian influenza viruses under selective pressure of antibody (in Chinese). Acta Microbiol Sin, 2009, 49: 955-959

9 Sun S H, Cui Z Z. Biological identification of Newcastle disease viruses isolated from eggs of a parent breeder farm (in Chinese). Acta Vet Zootech Sin, 2007, 38: 741-743

10 Venugopal K, Smith L M, Howes K, et al. Antigenic variants of J subgroup avian leucosis virus: Sequence analysis reveals multiple changes in env gene. J Gen Virol, 1998, 79: 757-766

11 Benns, Rutleddger T, Folks T, et al. Genomic heterogeneity of AIDS retroviral isolates from North America and Zaire. Science, 1985, 230: 949-951

12 Hwang S S, Boyle T J, Lyerly H K, et al. Identification of the envelope V3 loop as the primary determinant of cell tropism in HIV 1. Science, 1991, 253: 71-74

13 Shioda T, Levy L A, Chen M C. Small amino acid changes in the V3 hypervariable region of gp120 can affect the T-cell-line and macrophage tropism of human immunodeficiency virus type I. Proc Natl Acad Sci USA, 1992, 89: 9434-9438

14 Woelk C H, Jin L, Holmes E C, et al. Immune and artificial selection in the hemagglutinin $(\mathrm{H})$ glycoprotein of measles virus. J Gen Virol, 2001, 82: 2463-2474

15 Mast J, Nanbru C, Decaesstecker M, et al. Vaccination of chicken embryos with escape mutants of LaSota Newcastle disease virus induces a protective immune response. Vaccine, 2006, 24: 17561765

16 Qin Z M, Ma B C, Cui Z Z, et al. Newcastle disease virus heredity mutation and correlation of $H N$ and $F$ gene (in Chinese). Acta Microbiol Sin, 2006, 46: 227-232

17 Chambers P, Neabit M, Yusoff K, et al. Location of a neutralizing epitope for the hemagglutinin-neuraminidase glycoprotein of Newcastle disease virus. J Gen Virol, 1988, 69: 2115-2122

18 Iorio R M, Syddall R J, Sheehan J P, et al. Neutralization map of the hemagglutinin-neuraminidase glycoprotein of Newcastle disease virus: Domains recognized by monoclonal antibodies that prevent receptor recognition. J Virol, 1991, 65: 4999-5006

19 James J P, Elizabeth D S, Jeffrey J. Determination of the disulfide bond arrangement of Newcastle disease virus hemagglutinin neuraminidase. J Biol Chem, 2000, 275: 6469-6478

20 Sagrera A, Cobaleda C, Gonzalez De Buitrago J M, et al. Membrane glycoproteins of Newcastle disease virus: Nucleotide sequence of the hemagglutinin-neuraminidase cloned gene and structure function relationship of predicted amino acid sequence. Glycoconj J, 2001, 18: 283-289

Open Access This article is distributed under the terms of the Creative Commons Attribution License which permits any use, distribution, and reproduction in any medium, provided the original author(s) and source are credited. 